\title{
Different Evolution of Genotypic Resistance Profiles to Emtricitabine Versus Lamivudine in Tenofovir-Containing Regimens
}

\author{
Valentina Svicher, PhD, * Claudia Alteri, PhD, * Anna Artese, PhD, $\uparrow$ Federica Forbici, PhD, $\neq$ \\ Maria Mercedes Santoro, PhD, * Dominique Schols, PhD, $\S$ Kristel Van Laethem, PhD, $\S$ \\ Stefano Alcaro, PhD, $\uparrow$ Giosuè Costa $\uparrow$ Chiara Tommasi, MD, $\neq$ Mauro Zaccarelli, MD, $\neq$ \\ Pasquale Narciso, MD, $\neq$ Andrea Antinori, MD, $\neq$ Francesca Ceccherini-Silberstein, PhD, * \\ Jan Balzarini, MD, PhD, $\S$ and Carlo Federico Perno, MD, PhD*
}

Background: To investigate genotypic resistance profiles to emtricitabine + tenofovir (FTC + TDF) in-vivo and in-vitro, and compare them with lamivudine + tenofovir $(3 \mathrm{TC}+\mathrm{TDF})$.

Methods: Three hundred fifty-two HIV-1 B-subtype pol sequences from 42 FTC + TDF-treated patients, $403 \mathrm{TC}+\mathrm{TDF}$-treated patients, and 270 patients treated with $3 \mathrm{TC}$ plus another nucleoside reverse transcriptase inhibitor (but not TDF). All patients never received $\mathrm{FTC}$, 3TC, and TDF in their previous therapeutic regimen. $3 \mathrm{TC} / \mathrm{FTC} \pm \mathrm{TDF}$ resistance was investigated using in vitro selection experiments and docking simulations.

Results: The M184V mutation is less prevalent in FTC + TDFtreated patients than in 3TC + TDF-treated, and 3TC-treated/TDFnaive patients ( $14.3 \%$ versus $40.0 \%, P=0.01$ and $55.6 \%, P<0.001$ ).

Received for publication December 7, 2009; accepted March 8, 2010.

From the *Department of Experimental Medicine, University of Rome "Tor Vergata", Rome, Italy; $†$ Department of Pharmacobiological Sciences, University of Catanzaro "Magna Graecia", Roccelletta di Borgia (CZ), Italy; †I.N.M.I. "L. Spallanzani" Rome, Italy; and §Department of Microbiology and Immunology, Rega Institute for Medical Research, Katholieke Universiteit Leuven, Belgium.

Supported by grants from the Italian National Institute of Health, the Ministry of University and Scientific Research, Current and Finalized Research of the Italian Ministry of Health. It was also supported from the Concerted Actions of the K.U.Leuven (GOA no. 05/19) (to JB \& DS) and the "Fonds voor Wetenschappelijk Onderzoek Vlaanderen" (FWO no. G.0611.09), and from CHAIN, Collaborative HIV and Anti-HIV Drug Resistance Network, Integrated Project no.223131, funded by the European Commission Framework 7 Program.

Part of these data have been presented at the 16th Conference on Retroviruses and Opportunistic Infections, February 8-11, 2009, Montreal, Canada, as poster presentation; XVIII International HIV Drug Resistance Workshop: Basic Principles and Clinical Implications, June 9-12, 2009, Fort Myers, $\mathrm{FL}$, as oral presentation.

J.B. is co-inventor of tenofovir. The other authors of this article have no conflict of interest.

The authors V.S. and C.A. equally contributed to the work

Correspondence to: Valentina Svicher, PhD, Department of Experimental Medicine, University of Rome "Tor Vergata”, Via Montpellier 1, 00133 Rome, Italy (e-mail: valentina.svicher@uniroma2.it).

Supplemental digital content is available for this article. Direct URL citations appear in the printed text and are provided in the HTML and PDF versions this article on the journal's Web site (www.jaids.com).

Copyright () 2010 by Lippincott Williams \& Wilkins
Multivariable analysis shows that factors correlated with a lower probability of M184V emergence at failure were the use of FTC compared with 3TC [odds ratio (OR): 0.32 (95\% confidence interval (CI): 0.10 to 0.99$), P=0.04$ ], the use of boosted protease inhibitor, and the use of TDF [OR: 0.20 (95\% CI: 0.11 to 0.37$), \mathrm{P}<0.001$, and OR: 0.47 (95\%CI: 0.22 to 1.01$), P=0.05$, respectively]. In vitro selection experiments and docking analysis show that other reverse transcriptase (RT) mutations, even localized in RT connection domain, can be selected by $3 \mathrm{TC}+\mathrm{TDF}$ or FTC + TDF in M184V absence and can affect RT affinity for 3TC/FTC and/or TDF.

Conclusions: Our study shows lower rates of M184V development in FTC + TDF regimens versus 3TC + TDF and suggests a potential role of boosted protease inhibitors and TDF in delaying the M184V emergence. Novel RT mutational patterns, more complex than currently known, can contribute to $3 \mathrm{TC}, \mathrm{FTC}$, and TDF resistance.

Key Words: drug resistance, docking analysis, emtricitabine, HIV-1, in-vitro selection experiment, lamivudine, RT, structural analysis, tenofovir

\section{(J Acquir Immune Defic Syndr 2010;00:000-000)}

\section{INTRODUCTION}

Emergence of HIV-1 drug resistance is associated with increased mortality or faster progression of the HIV-1 infection. ${ }^{1-4}$ In this light, defining factors that can minimize the onset of drug resistance is crucial to maximize the opportunity to set up a long-term successful antiretroviral treatment. Recent studies have highlighted the protective role of boosted protease inhibitors (PIs) on the emergence of mutations associated with resistance not only to protease but also to reverse transcriptase (RT) inhibitors. ${ }^{5-7}$ In addition, another study has shown a lower emergence of the M184V mutations in patients receiving emtricitabine (FTC) + Tenofovir (TDF) + Efavirenz $(\mathrm{EFV})$ than in patients receiving Lamivudine (3TC) + azidothymidine $+\mathrm{EFV}^{8}$ These studies highlight the importance to identify those drug combinations that can prevent drug resistance emergence.

3TC and emtricitabine (FTC) are 2 structurally related nucleoside reverse transcriptase inhibitors (NRTIs), often 
proposed in combination with tenofovir (TDF) as NRTI backbone of antiretroviral regimens against HIV replication in both drug-naive and multiexperienced patients. ${ }^{9}$ Both $3 \mathrm{TC}$ and FTC usually select for the methionine-to-valine mutation in codon 184 (M184V) of RT. This mutation arises rapidly in failing therapy with 3TC and FTC and results in high-level (>100-fold) resistance to these drugs. ${ }^{10-12}$ This is often preceded by emergence of a methionine-to-isoleucine mutation in codon 184 (M184I), which is quickly replaced by M184V. ${ }^{13,14}$ Codon M184 is located in the highly conserved YMDD motif of RT, which is directly involved in binding the incoming nucleotide during reverse transcription. Although several studies have clarified the mechanism of action of the $\mathrm{M} 184 \mathrm{~V} / \mathrm{I}$ mutations, very few information is so far available regarding (1) the antiviral potential of 3TC or FTC when combined with TDF; (2) the factors modulating the rate of $\mathrm{M} 184 \mathrm{I} / \mathrm{V}$ emergence in vivo; (3) the role of other mutations (beyond $\mathrm{M} 184 \mathrm{I} / \mathrm{V}$ ) in resistance to FTC or 3TC when used not only as single drugs but also in combination with other antiretrovirals.

In the attempt to fulfill the above-mentioned gaps, the goal of this study is to define, by the analysis of clinical data supported by in-vitro experiments and modelling studies, characteristics and developments of genotypic resistance profiles to FTC + TDF versus 3TC + TDF.

\section{MATERIALS AND METHODS}

\section{Study Population}

Of 1370 HIV-1 B-subtype-infected patients failing a highly active antiretroviral therapy (HAART) regimen containing a $2^{\prime}$ deoxy-cytidine analogues (3TC or FTC), 352 patients treated with FTC + TDF $(\mathrm{n}=42), 3 \mathrm{TC}+\mathrm{TDF}(\mathrm{n}=40)$, and 3TC plus another NRTI (but not TDF) $(\mathrm{n}=270)$ were selected for this study. The following inclusion criteria were used: starting of HAART after 2000; no exposure to 3TC, FTC, and TDF in previous antiretroviral strategies; absence of $\mathrm{M} 184 \mathrm{~V}$ in a prior genotypic resistance test. Among 352 patients, 271 patients, distributed as follows, failed their first-line HAART regimen: $39 \mathrm{FTC}+\mathrm{TDF}-$ treated patients, $293 \mathrm{TC}+\mathrm{TDF}-$ treated patients, and $2033 \mathrm{TC}+$ another NRTI (but not TDF) patients. Virological failure was defined as 2 consecutive determinations of HIV-1 RNA $>50$ copies per milliliter. All patients enrolled give written informed consent. Data for all patients were stored in a specifically designed anonymous database that included genotypic, demographic, immunologic, virologic, and therapeutic parameters.

\section{HIV Sequencing}

HIV genotype analysis was performed on plasma samples by means of a commercially available kit (ViroSeq HIV-1 genotyping system; Abbott Laboratories, Abbott Park, IL). Briefly, RNA was extracted, retrotranscribed by murine leukemia virus RT, and amplified with Amplitaq-Gold polymerase enzyme by using 2 different sequence-specific primers for 40 cycles. Pol-amplified products (containing the entire protease and the first 335 amino acids of the RT open reading frame) were full-length sequenced in sense and antisense orientations by an automated sequencer (ABI 3100) by using 7 different overlapping sequence-specific primers. ${ }^{15}$
Sequences having a mixture of wild-type and mutant amino acid residues at single positions were considered to have the mutant(s) at that position.

\section{Statistical Analysis}

1. Mutation prevalence: The prevalence of mutations associated with $3 \mathrm{TC}$ and FTC resistance was calculated in isolates from patients failing their first HAART regimen containing FTC + TDF $(\mathrm{n}=42), 3 \mathrm{TC}+\mathrm{TDF}(\mathrm{n}=40)$, and $3 \mathrm{TC}+$ another NRTI but not TDF $(n=270) . \chi^{2}$ tests of independence were used to verify whether the differences in frequency between the different groups of patients were statistically significant.

2. Factor affecting the presence of M184V at failure: A multivariable logistic regression was performed to define factors positively and negatively correlated with the presence of M184V at 3TC/FTC failure. The following variables were considered: subject demographic, year of treatment, use of cytidine analogues, drugs co-administered with 3TC/FTC, viremia and CD4 cell count at baseline, years under treatment, number of previous treatment failures, number of drugs received in the past, previous exposure to nonnucleoside reverse transcriptase inhibitor (NNRTI), previous exposure to NNRTI. The analysis was performed in the whole cohort of 352 HAART-treated patients, and in the subset of 270 patients receiving $3 \mathrm{TC} \pm \mathrm{TDF}$.

\section{In Vitro Selection Experiments}

In a 48-well tray, $10^{5} \mathrm{CEM}$ cells were seeded in $1 \mathrm{ml}$ RPMI-1640 culture medium-containing $10 \%$ fetal calf serum and $2 \mathrm{mM}$ L-glutamine. TDF [ $(R)$-PMPA] (at 10, 5, 2.5, 1.25 $\mu \mathrm{M}$ ), FTC (at $0.2,0.1,0.05$ and $0.025 \mu \mathrm{M}$ ) or $3 \mathrm{TC}$ (at $0.2,0.1$, 0.05 and $0.025 \mu \mathrm{M}$ ), and combinations of TDF with FTC or with $3 \mathrm{TC}$ were added to the wells. Then, $50 \mu \mathrm{L}$ of $\mathrm{HIV}-1\left(\mathrm{III}_{\mathrm{B}}\right)$ $(190,000 \mathrm{pg}$ p24) was added and the cultures further incubated for 4-5 days. At each subcultivation, 25-50 $\mu \mathrm{L}$ cell suspension was transferred to fresh cell cultures containing the same fixed concentrations of the test compounds. After the 10th subcultivation ( 2 months), microscopically visible cytopathicity (CPE) and p24 (pg/mL/well) measurements for all subcultures were determined. The cultures were thereafter further subcultivated for 4 additional passages in the absence of the compounds to verify potential virus breakthrough. ${ }^{16}$ The methodology of RT sequencing is reported in Snoeck et $\mathrm{al}^{17}$.

\section{Association With 3TC, FTC, and TDF Susceptibility}

We analyzed genotype-phenotype correlations from the HIV Stanford Drug Resistance Database ${ }^{18}$ to assess the association of mutations with 3TC, FTC, and TDF susceptibility. In particular, for each drug, we compared the median changes in resistance (n-fold) in relation to sequences with or without specific mutation. The change in resistance (n-fold) was measured by Virco Antivirogram assay. Mann-Whitney test was used to assess statistically significant difference.

\section{Structural and Docking Analysis}

The X-ray crystallographic coordinates of RT complexed to DNA deposited in the Protein Data Bank ${ }^{19}$ with code $1 \mathrm{RTD}^{20}$ were used for the structural analysis. For the 3 
inhibitors, 3TC, TDF, and FTC, the heterocyclic base was appropriately modified before docking into the enzyme. Also the heterocyclic moiety of the $n+1$ th nucleotide in template overhang was modified according to the base complementary with respect to the incoming NRTI. Thus, in 3TC-TP and FTC-TP complexes, the adenine moiety, which was in the original X-ray structure, was changed into guanine, whereas in TDF-TP modeling, it was modified into thymine. Finally, the NRTI triphosphate was manually located in such an orientation paired with its complementary base in the template strand. From this starting model, the analyzed mutants were generated by single-residue replacement in both chains and energy minimized using the united atom AMBER* force field ${ }^{21}$ and the GB/SA water implicit solvation model ${ }^{22}$ as implemented in MacroModel ver 7.2 (Schrödinger). ${ }^{23}$

The fully optimized receptor coordinates were used for the docking simulations, carried out by AutoDock version $4 .^{24}$ The map box was fixed equal to $70.190 \AA^{3}$, AMBER was used as force field, the receptor was kept rigid, and the drug flexible. This method applied a Lamarckian model of genetics, generating 50 allowed configurations per ligand. We carried out the maps for A, C, HD, N, NA, OA, P, SA, and F probes; the dielectric constant was set equal to 80 .

To select the AutoDock generated poses able to receive the nucleophilic attack by the free $3^{\prime} \mathrm{OH}$ of the DNA template
(NRTI-template distance), a distance cut-off equal to $6.0 \AA$ was adopted and the best pose, as ranked by the free-energy value, was identified.

All 3dimensional figures were performed using PyMOL graphics and modeling package ver $0.98 .^{25}$

\section{RESULTS}

\section{Patients Characteristics}

Three hundred fifty-two HIV-1 B-subtype-infected patients treated with FTC + TDF $(\mathrm{n}=42)$, 3TC + TDF $(\mathrm{n}=40)$, and 3TC plus other NRTI (but not TDF) $(\mathrm{n}=270)$ met the inclusion criteria for this study (Table 1). All patients have started treatment after 2000 (year of introduction of TDF in clinical practice); none of them was receiving a mono or dual therapy at the time of resistance testing, and none of them received 3TC, FTC, and TDF in their previous therapeutic regimens. These 3 groups of patients are also comparable in terms of the median baseline viremia and CD4 cell count, of the median change of viremia and CD4 cell count from baseline to failure, of the median time spent under virological failure, and of the percentage of patients treated with PIs or NNRTIs (Table 1).

\section{TABLE 1. Patient Characteristics}

\begin{tabular}{|c|c|c|c|c|c|}
\hline & \multicolumn{3}{|c|}{ Treatment With } & \multirow[b]{2}{*}{$P^{*}$} & \multirow[b]{2}{*}{$P \dagger$} \\
\hline & FTC + TDF & $3 T C+T D F$ & 3TC (Naive to TDF) & & \\
\hline Number of patients failing treatment & 42 & 40 & $270 \ddagger$ & - & - \\
\hline Patients, $\mathrm{n}(\%)$ failing their first-line treatment & 39 & 29 & 203 & - & - \\
\hline Male, N (\%) & $32(76.2)$ & $24(64.9)$ & $191(74.3)$ & NS & NS \\
\hline HIV exposure, n (\%) & $32(76.2)$ & $24(64.9)$ & $191(74.3)$ & - & - \\
\hline MSM & $11(33.3)$ & $6(18.2)$ & $37(21.5)$ & NS & NS \\
\hline Age (yrs), median (IQR) & $40(33-48)$ & $43(36-48)$ & $40(35-45)$ & NS & NS \\
\hline \multicolumn{6}{|l|}{ Viremia (log copies $/ \mathrm{mL})$, median (IQR) } \\
\hline Baseline & $5.2(4.2-5.5)$ & $5.0(4.6-5.5)$ & $5.1(4.6-5.4)$ & NS & NS \\
\hline Change from baseline to failure & $-4.2(-5.7$ to -1.1$)$ & $-4.7(-5.6$ to 3.5$)$ & $-4.9(-5.5$ to -4.5$)$ & NS & NS \\
\hline \multicolumn{6}{|l|}{ CD4 cell count (cells/uL), median (IQR) } \\
\hline Baseline & $139(89-304)$ & $153(42-380)$ & $168(63-311)$ & NS & NS \\
\hline Boosted PI & $21(50.0)$ & $16(40.0)$ & $135(50.0)$ & NS & NS \\
\hline Number of previous HAART regimens, median (IQR) & $5(7-12)$ & $7(5-12)$ & $5(4-8)$ & NS & NS \\
\hline \multicolumn{6}{|l|}{ Number of previously received drugs, median (IQR) } \\
\hline NRTI & $4(2-4)$ & $4(2-6)$ & $2(2-4)$ & NS & NS \\
\hline NNRTI & $1(0-2)$ & $1(0-1)$ & $0(0-1)$ & NS & NS \\
\hline PI & $2(1-3)$ & $1(1-2)$ & $0(0-1)$ & NS & NS \\
\hline
\end{tabular}

*Statistically significant difference between FTC + TDF-treated patients and 3TC + TDF-treated patients was assessed by Fisher exact test for categorical data and by Wilcoxon test for continuous variables.

$\dagger$ Statistically significant differences between 3TC + TDF-treated patients and 3TC- but not TDF-treated patients were assessed by Fisher exact test for categorical data and by Wilcoxon test for continuous variables.

\$No patients received $3 \mathrm{TC}$ mono or dual therapy. 


\section{Prevalence of M184V at Virological Failure In Vivo}

Genotypic analysis showed a significantly lower M184V prevalence in patients failing FTC + TDF than in patients failing $3 \mathrm{TC}+\mathrm{TDF}$, and in patients failing $3 \mathrm{TC}$ plus another NRTI (but not TDF) [6 of $42(14.3 \%)$ versus 16 of $40(40.0 \%)$ versus 150 of $270(55.6 \%), P=0.01$ and $P<0.001$, respectively] (Fig. 1). This result was confirmed when the analysis was restricted to the subset of patients failing first-line HAART [5 of $39(12.8 \%)$ versus 10 of $29(34.5 \%)$ versus 104 of $203(51.2 \%), P=0.04$ and $P<0.001$, respectively] (data not shown).

Multivariable analysis confirms that the use of FTC is an independent factor significantly correlated with a lower probability of $\mathrm{M} 184 \mathrm{~V}$ emergence at failure than 3TC [odds ratio (OR): 0.32 (95\% confidence interval (CI): 0.10 to 0.99 ), $P=0.04$ ] (Table 2). Other factors independently correlated with a lower probability of M184V emergence at 3TC/FTC failure were the use of boosted PIs [OR: 0.19 (95\% CI: 0.10 to $0.37), P<0.001$, compared with NNRTI], and the use of TDF [OR: 0.47 (95\% CI: 0.21 to 1.01$), \mathrm{P}=0.05$, compared with thymidine analogues] (Table 2). The use of TDF significantly correlated with a lower M184V emergence compared with abacavir only at univariable analysis [OR: 0.29 (95\% CI: 0.12 to 0.69$), P=0.004]$.

The negative correlation of boosted PIs and TDF with M184V emergence is confirmed when the analysis was restricted only to patients receiving $3 \mathrm{TC} \pm \mathrm{TDF}$ [OR: 0.20 (95\% CI: 0.10 to 0.38$), P<0.001$, and OR: $0.47(95 \%$ CI: 0.21 to 1.01 ), $P=0.05$ ] (data not shown).

\section{In Vitro Selection Experiments of Mutations Associated With Resistance to 3TC/FTC and TDF}

\section{Activity of FTC, 3TC, and TDF (as Single Compounds or in Combination) in Suppressing HIV-1 Replication}

TDF and 3TC or FTC were administered to CEM cell cultures exposed to a high input of HIV-1(III $)(190,000$ $\mathrm{pg}$ of p24). Under these experimental conditions, TDF, FTC, and 3TC inhibited the virus-induced $\mathrm{CPE}$ at an $\mathrm{EC}_{50}$ (50\% effective concentration) of $\sim 10 \mu \mathrm{M}, 0.075 \mu \mathrm{M}$, and $0.20 \mu \mathrm{M}$, respectively (Supplemental Digital Content 1, http://links.lww.com/QAI/A74). This is in line with previously published data. ${ }^{16,26}$

Serial subcultivations of all drug-treated virus-infected cell cultures were then performed by keeping the (combination of) drug concentrations unaltered in the new cell cultures. After 10 subcultivations (4-5 days for each subcultivation) each culture was examined for viral cytopathicity and p24 content. Interestingly, only 2 of 16 different 3TC + TDF combinations had resulted in no cytopathicity and no p24 (virus-free cultures) (TDF $10 \mu \mathrm{M}$ in combination with 3TC $0.2 \mu \mathrm{M}$ and $0.1 \mu \mathrm{M})$. By contrast, 7 of 16 different FTC + TDF combinations resulted virus-free (FTC $0.1 \mu \mathrm{M}$ in combination with TDF $10 \mu \mathrm{M}, 5 \mu \mathrm{M}$, and $2.5 \mu \mathrm{M}$, and FTC $0.2 \mu \mathrm{M}$ in combination with TDF $10 \mu \mathrm{M}, 5 \mu \mathrm{M}$, and $2.5 \mu \mathrm{M}$, and $0 \mu \mathrm{M}$ ) (Supplemental Digital Content 1,

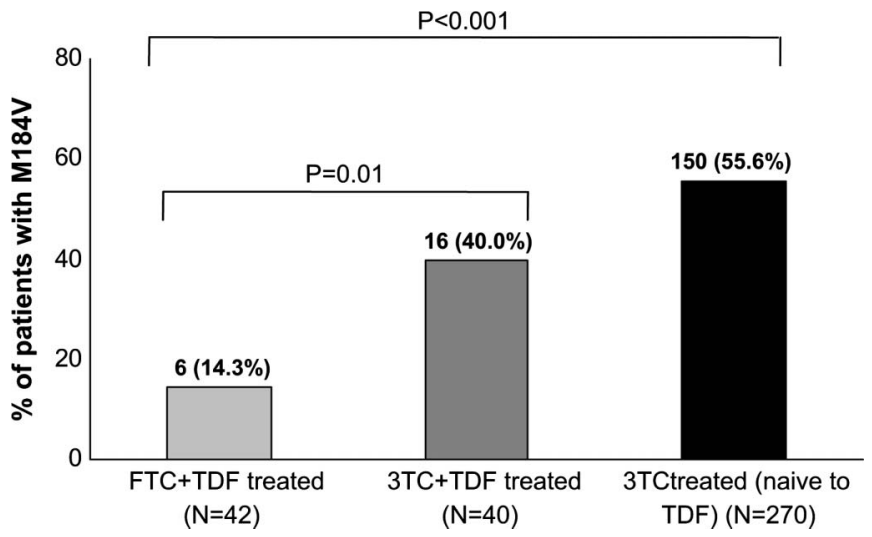

FIGURE 1. Prevalence of M184V in patients failing FTC + TDFcontaining HAART (light grey bars), 3TC + TDF-containing HAART (dark grey bars), and 3TC-containing HAART (black bars). Statistically significant differences were assessed by Fisher exact test.

http://inks.lww.com/QAI/A74) as confirmed by lack of p24 detection (Supplemental Digital Content 2, http://links.lww.com/QAI/A75). The microscopical reading fully correlated with the p24 measurements. In the p24negative cultures, no viral DNA could be amplified. In addition, for 5 of 7 p24-negative cultures under treatment with FTC + TDF, the same concentrations of 3TC + TDF correlated with a viral production $>450 \mathrm{pg} / \mathrm{mL}$.

Also, it should be noticed that combination of several fixed drug concentrations proved clearly superior in suppressing virus replication when compared with the use of 2-fold higher concentrations of the single drugs. For example, single administration of $0.05 \mu \mathrm{M}$ FTC and $5 \mu \mathrm{M}$ TDF did not result in any viral suppression (after the tenth subcultivation) (p24: $>1000 \mathrm{pg} / \mathrm{mL}$ ), whereas the combination of these drug concentrations $(0.05 \mu \mathrm{M}$ FTC $+5 \mu \mathrm{M}$ TDF) markedly suppressed virus replication (p24: $2.07 \mathrm{pg} / \mathrm{mL}$ ). Double single-drug concentrations (ie, FTC at $0.1 \mu \mathrm{M}$ or TDF at $10 \mu \mathrm{M}$ ) allowed virus replication at 818.98 and $540.54 \mathrm{pg} / \mathrm{mL}$, respectively.

Thus, the check-board combinations revealed that although FTC was only slightly superior to 3TC alone in suppressing a high virus load as a single compound, the combinations of FTC + TDF were superior to the combinations of $3 \mathrm{TC}+\mathrm{TDF}$ in suppressing virus replication and rendering the HIV-exposed cell cultures free of virus (significance calculated by a Student $t$ test, with a 2-tailed distribution: $P<0.0005$ ).

\section{Genotypic Analysis}

Analysis of the RT gene was performed on all cell cultures showing the presence of HIV replication (detectable CPE and p24). The different combinations of FTC + TDF never selected for the M184I or M184V mutation (Fig. 2). The M184I mutation was observed only under the selective pressure of FTC when used as single compound without TDF (Fig. 2; FTC $0.1 \mu \mathrm{M}$ ). In the case of 3TC + TDF, M184I was selected when $3 \mathrm{TC}$ was combined with the lowest concentration of TDF (1.25 $\mu \mathrm{M}$, about 8-fold lower than the TDF EC50) 
TABLE 2. Odds Ratio for the Emergence of the M184V Mutation from Fitting a Multivariable Logistic Regression Model in Patients Failing 3TC/FTC Treatment

\begin{tabular}{|c|c|c|c|c|}
\hline \multirow{2}{*}{$\begin{array}{c}\text { Independent Predictors of the M184V } \\
\text { Presence at 3TC/FTC Failure }\end{array}$} & \multicolumn{2}{|c|}{ Univariate Analysis } & \multicolumn{2}{|c|}{ Multivariate Analysis } \\
\hline & Odds Ratio $(95 \%$ CI) & $P$ & Odds Ratio (95\% CI) & $P$ \\
\hline \multicolumn{5}{|l|}{ Sex } \\
\hline Male versus female & $1.02(0.61$ to 1.71$)$ & 0.94 & - & - \\
\hline \multicolumn{5}{|l|}{ Transmission route } \\
\hline Heterosexual & 1 & - & - & - \\
\hline CD4 cell count at baseline (cells/uL) & $1.00(0.99$ to 1.01$)$ & 0.79 & - & - \\
\hline Viremia at baseline (log copies/mL) & $1.00(1.00$ to 1.00$)$ & 0.56 & - & - \\
\hline \multicolumn{5}{|l|}{ Use of cytidine analogues } \\
\hline FTC versus $3 \mathrm{TC}$ & $0.15(0.06$ to 0.36$)$ & $<0.001$ & $0.32(0.10$ to 0.99$)$ & 0.04 \\
\hline \multicolumn{5}{|c|}{ Administration of $3 \mathrm{TC} / \mathrm{FTC}$ with another NRTI } \\
\hline TDF versus TA & $0.24(0.13$ to 0.43$)$ & $<0.001$ & $0.47(0.21$ to 1.01$)$ & 0.05 \\
\hline Time under treatment, weeks & $0.73(0.30$ to 1.76$)$ & 0.49 & - & - \\
\hline Number of previous regimens & $0.88(0.75$ to 1.03$)$ & 0.10 & - & - \\
\hline Previously received drugs, $\mathrm{N}$ & $0.89(0.77$ to 1.04$)$ & 0.99 & - & - \\
\hline Previous use of NNRTI & $1.31(0.41$ to 4.16$)$ & 0.65 & - & - \\
\hline Previous use of PI & $1.39(0.38$ to 5.07$)$ & 0.61 & - & - \\
\hline
\end{tabular}

The analysis was performed on 352 patients. All predictor variables considered for the analysis were reported. Multivariable analysis was performed by using variables that resulted significant in the univariable model. DDI was not included in the analysis because it was received in $<5 \%$ of patients.

$\mathrm{TA}$, thymidine analogue; $\mathrm{ABC}$, abacavir.

(Fig. 2). No M184I/V emergence at all was observed by the other combinations of $3 \mathrm{TC}+\mathrm{TDF}$ and FTC + TDF.

Other RT mutations were selected in-vitro under the selective pressure of $3 \mathrm{TC}+\mathrm{TDF}$ or FTC + TDF in the absence of M184V/I. In particular, 3TC + TDF selected for the pair of mutations T69I + P345S and also for mutations P176A, H361Y, and S379G, whereas FTC + TDF selected for the pair of mutations P19S + E169K and for T472I and N494S (Fig. 2). Different mutations were selected by TDF when used without 3TC or FTC: S162N, F214L, and K385N (localized in the connection domain of HIV-1 RT) (Fig. 2). No K65R was found in these cell cultures.

We then investigated the role of mutations detected in in vitro selection experiments by analyzing sequences with known NRTI susceptibility (measured by Virco Antivirogram assay) from the Stanford HIV Drug Resistance Database. We found that the presence of T69I (in the absence of M184V and TAMs) significantly correlated with a 63.3 -fold $(P=0.03)$ and 37.8-fold $(P=0.04)$ increase in 3TC and FTC resistance, respectively (Table 3). This mutation is also associated with a 14.4-fold increase in TDF resistance (though not statistically significant).

\section{Docking Analysis}

Based on the above-mentioned results, we further investigated the role of T69I in resistance to 3TC, FTC, and TDF by molecular modeling. We also used this approach to characterize the role of $\mathrm{P} 345 \mathrm{~S}$ in resistance to the drugs because this mutation was coselected (in the same viral genome) together with the T69I during the in vitro selection experiments.

After our previously reported approach, ${ }^{27}$ we applied a distance-filtering criterion to analyze the docking results, assuming a major "reagent-like" control in the NRTI mechanism of action. A distance $>6 \AA$ was considered to be not compatible with the binding of the drug with RT.

After docking simulations, we found that the distance between 3TC and the oxydrilic group of the nascent DNA chain is increased $(>6 \AA)$ in the presence of T69I, likely hampering the affinity between the RT and the nucleotide analogue (Fig. 3). Conversely, P345S, alone or in combination with T69I, did not abolish the nucleophilic attack between 3TC and the nascent DNA chain but was associated with an unfavorable binding affinity $(\Delta \mathrm{G}$ : $-2.74 \mathrm{Kcal} / \mathrm{mol}$ for wild-type, $-1.85 \mathrm{Kcal} / \mathrm{mol}$ for P345S, $-2.28 \mathrm{Kcal} / \mathrm{mol}$ for T69I + P345S).

Similarly, the presence of either T69I or P345S caused a larger distance between the upcoming TDF and the oxydrilic group of the nascent DNA chain (distance $>6 \AA$ ), with a further increase when these 2 mutations were combined (Fig. 4). Such a result was also confirmed by the energetic analysis of the best poses, that, in presence of both substitutions, were always incompatible with the bond formation and correlated with an unproductive energetic profile $(\Delta \mathrm{G}:-0.88 \mathrm{Kcal} / \mathrm{mol}$ for wild-type, $+0.15 \mathrm{Kcal} / \mathrm{mol}$ for T69I, $+1.05 \mathrm{Kcal} / \mathrm{mol}$ for P345S, $-0.25 \mathrm{Kcal} / \mathrm{mol}$ for T69I + P345S). 
FIGURE 2. Amino acid mutations in the RT of HIV-1 present after 10 invitro passages in different checkboard combinations with drug concentrations ranging $0-10 \mu \mathrm{M}$ for TDF, 0-0.05 $\mu \mathrm{M}$ for FTC, and $0-0.2 \mu \mathrm{M}$ for 3TC.

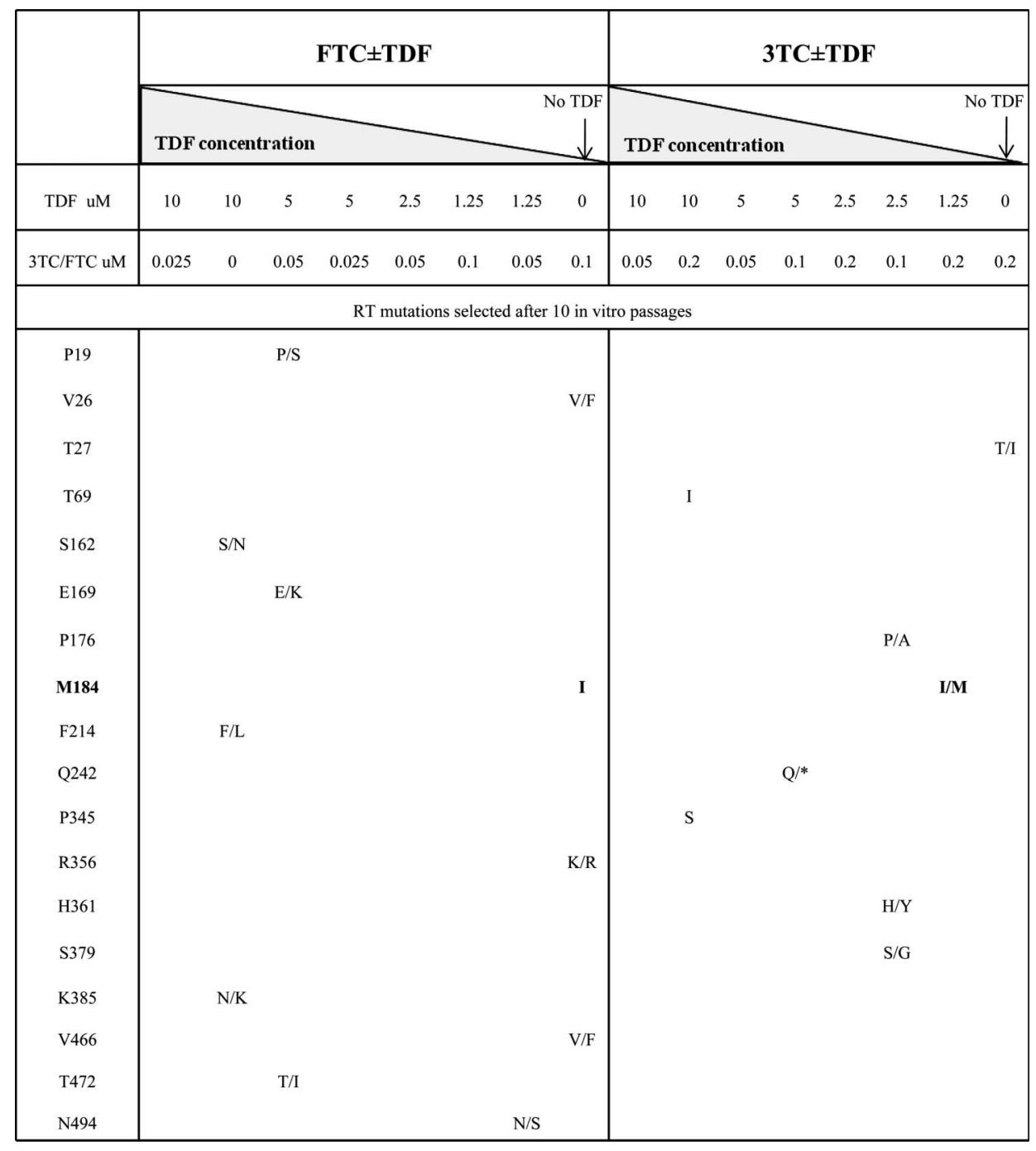

Finally, in the FTC/RT complexes, the copresence of T69I + P345S correlated with an increased distance between FTC and the oxydrilic group of DNA and with a reduced binding affinity with respect to the WT sequence (data not shown). When present as a single mutation, P345S correlated only with an unfavorable energetic profile, whereas T69I did not seem to negatively affect FTC binding $(\Delta \mathrm{G}$ : -2.74 $\mathrm{Kcal} / \mathrm{mol}$ for wild-type, $-3.24 \mathrm{Kcal} / \mathrm{mol}$ for T69I, -2.02 $\mathrm{Kcal} / \mathrm{mol}$ for $\mathrm{P} 345 \mathrm{~S},-2.19 \mathrm{Kcal} / \mathrm{mol}$ for T69I + P345S).

Overall results support the role of T69I and P345S, alone or in combination, in resistance to the cytidine analogues 3TC and FTC and to TDF.

\section{DISCUSSION}

This study shows that the use of FTC significantly correlates with a decreased probability of M184V emergence at HAART failure compared with the use of 3TC. This result, confirmed both in univariable and multivariable analysis, is fully consistent with a previous study showing a lower occurrence of M184I/V in patients who started FTC + TDF +
EFV compared with those who received $3 \mathrm{TC}+\mathrm{ZDV}+\mathrm{EFV}^{8}$ Our result has been also confirmed in an independent dataset showing that virological rebound in patients treated with 3TC/TDF plus a NNRTI or a PI (with or without ritonavir) is associated with a higher probability of developing 3 NRTI resistance mutations including the M184V and 1 NNRTIassociated mutation when compared with individuals receiving FTC + TDF. $^{28}$

The lower prevalence of M184V can be explained by the higher potency of FTC than 3TC, as suggested by the present and previous in vitro studies ${ }^{10,29}$ and/or by the different intracellular pharmacokinetics of FTC triphosphate versus 3TC triphosphate. ${ }^{30}$ Indeed, FTC triphosphate has been shown to have a $>2$-fold higher intracellular half-life than 3TC triphosphate. ${ }^{31,32}$ This is due to the ability of FTC to inhibit the activity of the cellular efflux proteins, such as the multidrug resistance-associated proteins, that extrude the drugs out of the CD4+ cells. ${ }^{33}$ The higher intracellular half-life of FTC can increase its ability to inhibit the RT enzyme, thus reducing the probability of emergence of the M184V mutation. In addition to this, the lower prevalence of M184V may be also related to 
TABLE 3. Association of T69I With Decreased Susceptibility to 3 TC, FTC, and TDF

\begin{tabular}{lcccc}
\hline $\begin{array}{c}\text { Drug } \\
\text { Susceptibility }\end{array}$ & Mutation & $\begin{array}{c}\text { Number of } \\
\text { Sequences* }\end{array}$ & $\begin{array}{c}\text { Fold Change } \\
\text { (Median, IQR) } \dagger\end{array}$ & $\begin{array}{c}\text { Fold Change } \\
\text { Increase }\end{array}$ \\
\hline 3TC & $69 \mathrm{~T}$ & $192(98.97)$ & $0.9(0.5-1.4)$ & $63.3 \ddagger$ \\
& $69 \mathrm{I}$ & $2(1.03)$ & $95.2(84.9-105.6)$ & \\
FTC & $69 \mathrm{~T}$ & $58(98.30)$ & $0.9(0.4-1.9)$ & $37.8 \S$ \\
& $69 \mathrm{I}$ & $1(1.70)$ & 71.9 & \\
TDF & $69 \mathrm{~T}$ & $79(98.75)$ & $1.0(0.7-1.6)$ & 14.4 \\
& 69I & $1(1.25)$ & 23.3 & \\
\hline
\end{tabular}

*Sequences and phenotypic data were collected from Stanford HIV Drug Resistance Database (http://hivdb.stanford.edu). Sequences do not contain M184V and thymidine analogues mutations.

$\dagger$ The resistance values ( $\mathrm{n}$-fold) are derived from the real phenotypic data measured in vitro by Virco Antivirogram assay.

\$The difference in 3TC resistance between 69I and 69T is statistically significant $(P=$ 0.03 , Mann-Whitney test).

§The difference in FTC resistance between 69I and 69T is statistically significant $(P=0.043$, Mann-Whitney test).

IQR, interquartile range.

the coformulation of FTC + TDF that could favor the adherence of the patients to the therapeutic regimen.

Our multivariable model also shows that the use of boosted PIs leads to lower rates of M184V emergence at the failure of $2^{\prime}$-deoxycytidine analogue-containing HAART regimen. This is consistent with previous studies highlighting a lower emergence of mutations associated with resistance to both protease and RT inhibitors at the failure of a boosted PIcontaining HAART. ${ }^{5-7}$ In particular, the negative correlation between the use of boosted PIs and the emergence of M184V shown in our study is fully consistent with Gupta et $\mathrm{al}^{7}$ showing a lower occurrence of M184V in patients who started boosted PI-based HAART compared with those who received a NNRTI regimen $(21.0 \%$ versus $35.3 \%, P<0.001)$.

In addition, our study suggests a potential role of tenofovir in delaying the emergence of M184V at 3TC failure compared with the use of thymidine analogues. Although this correlation is less strong than that observed for boosted PIs, and needs further confirmation in larger cohort of patients, it is consistent with previous studies showing the complete deselection of the M184V mutation during the pressure imposed by tenofovir in vitro selection experiments. ${ }^{34}$ Another study also showed the reversion of the M184V in rhesus macaques within 24 weeks of treatment with TDF, even in the presence of $3 \mathrm{TC}$ or FTC. ${ }^{35}$ The negative correlation between TDF and the M184V detection at 3TC/FTC failure may be explained by the fact that the M184V mutation has been shown to confer hypersusceptibility to TDF. ${ }^{36}$ Thus, the co-administration of a 2' deoxycytidine analogue with TDF may potentially delay the emergence of viral strains that failed by the M184V mutation. Further studies are necessary to verify this hypothesis.

Thus, overall findings highlight the importance to identify those drug combinations that can minimize drug resistance to HAART. Understanding this point is crucial to maximize the opportunity for successful and subsequent therapies after viral rebound not only in the western countries but also in resource-limited settings.
FIGURE 3. Insight of the (A) wildtype, (B) T69l, (C) P345S, and (D) T69I + P345S 3TC RT-template complex showing the distance between the drug and the free $3^{\prime} \mathrm{OH}$ of the DNA template. The RT and the DNA are shown, respectively, as lime and orange cartoon; 3TC and the nucleotide in $3^{\prime}$ are represented as grey carbon sticks. The distances, expressed in $\AA$, are indicated as yellow dashed lines. A distance higher than $6 \AA$ was considered to be not compatible with the binding of the drug with the RT.
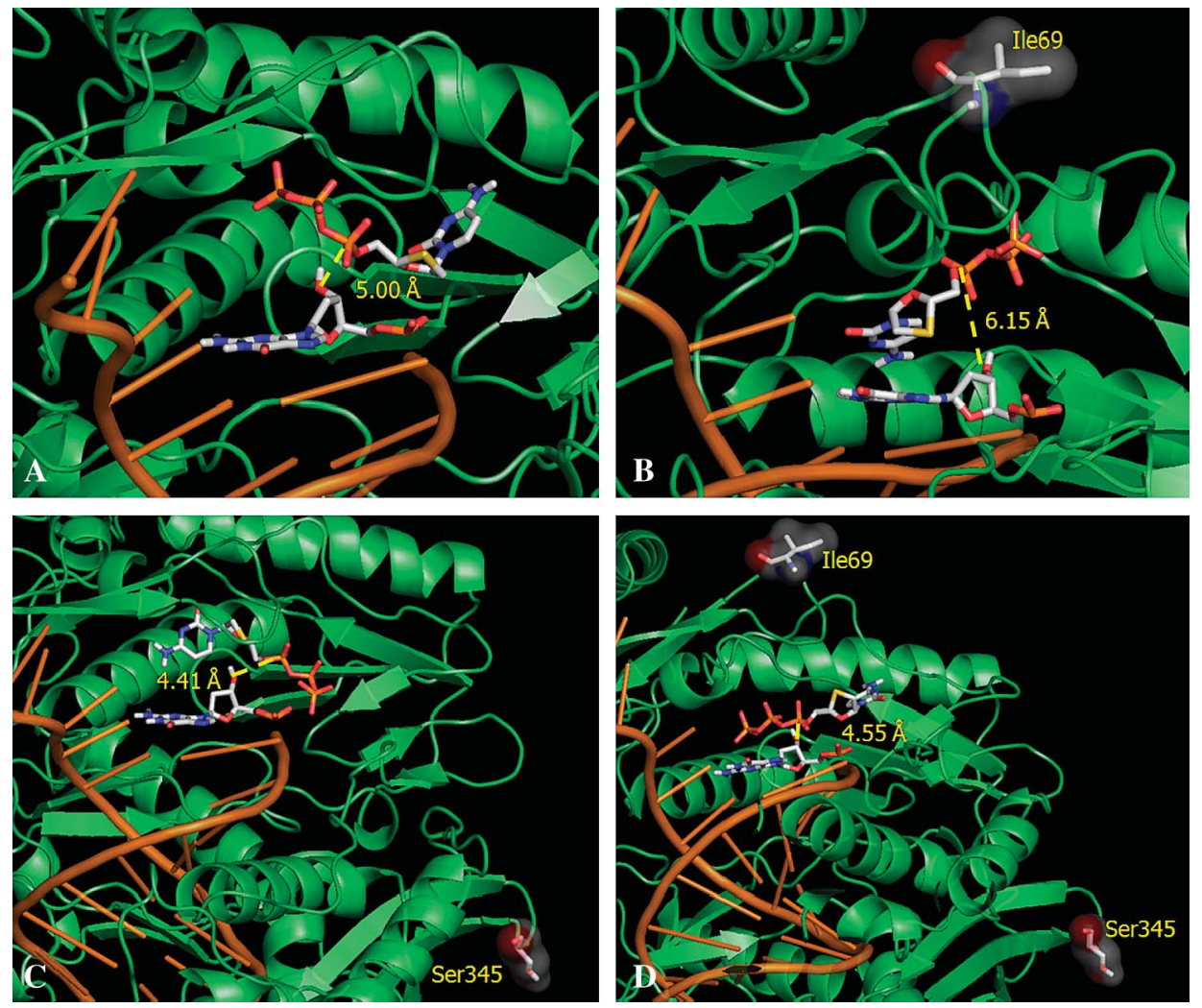

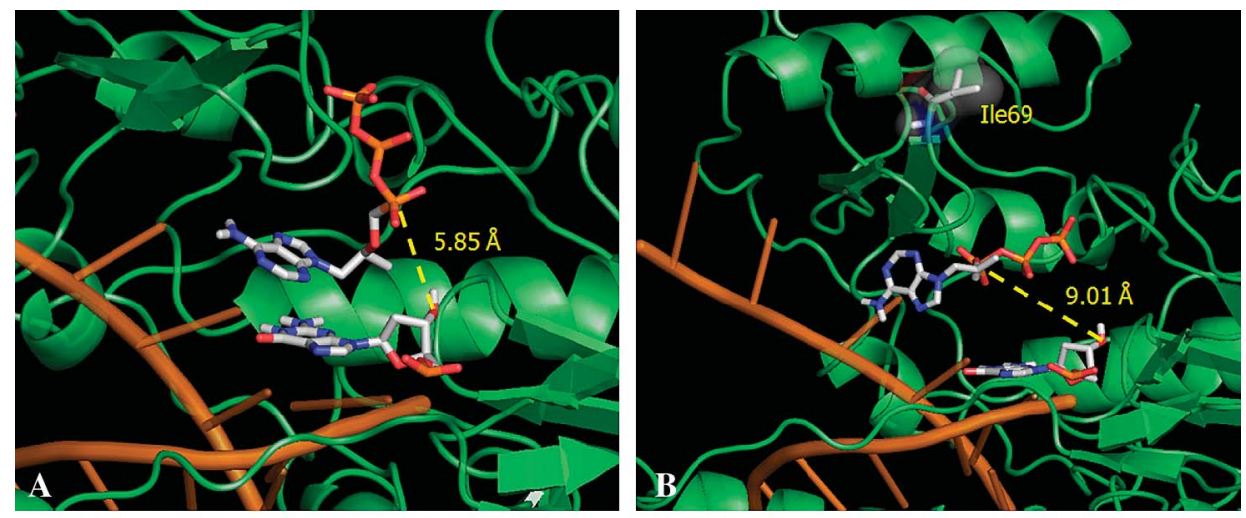

FIGURE 4. Insight of the (A) wildtype, (B) T69l, (C) P345S, and (D) T69I + P345S TDF RT-template complex showing the distance between the drug and the free $3^{\prime} \mathrm{OH}$ of the DNA template. The RT and the DNA are shown, respectively, as lime and orange cartoon; TDF and the nucleotide in $3^{\prime}$ are represented as grey carbon sticks. The distances, expressed in $\AA$, are indicated as yellow dashed lines. A distance higher than $6 \AA$ was considered to be not compatible with the binding of the drug with the RT.
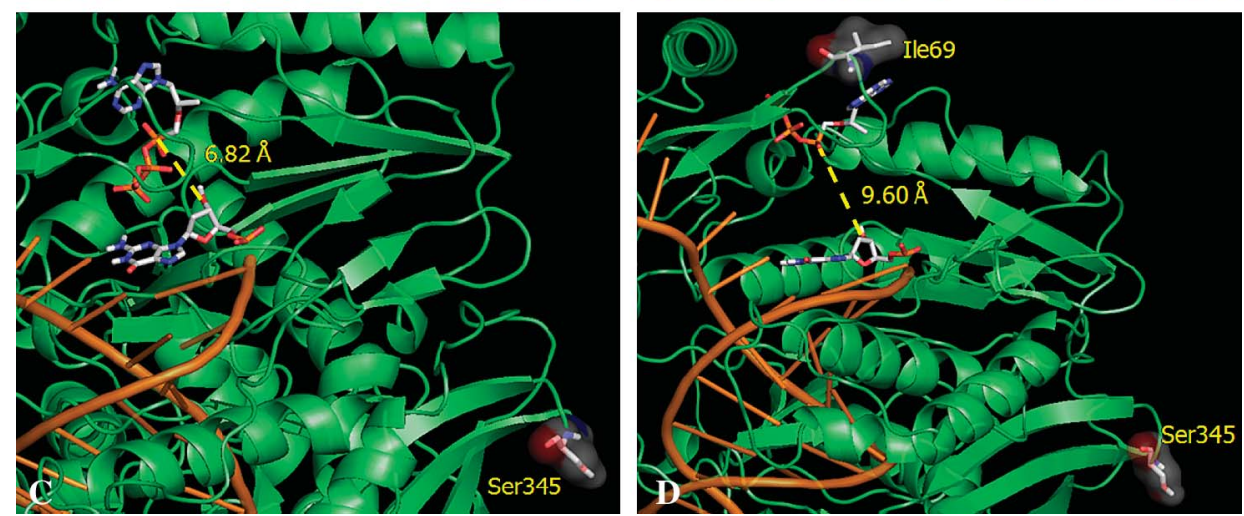

Furthermore, this is the first in vitro study showing that although FTC was in vitro only slightly superior to 3TC alone in suppressing a high virus load as a single compound, the combinations of FTC + TDF were clearly superior to the combinations of 3TC + TDF (statistical analysis: $P<0.0005$ ). This is consistent with the synergism of FTC and TDF reported by previous biochemical studies. In particular, it has been demonstrated that the levels of both TDF diphosphate and FTC triphosphate were significantly higher when these 2 drugs are combined compared with the levels seen with the individual drugs. ${ }^{37}$ Another recent study showed that the presence of FTC triphosphate can enhance the formation of the dead-end complexes by HIV-1 RT and TDF-terminated DNA, thus resulting in a reduced terminal NRTI excision. ${ }^{38}$

This study also highlights the appearance of novel mutations involved in resistance to the cytidine analogues 3TC and FTC and also to TDF. This is the case for T69I (occurring in $0.5 \%$ of drug-experienced and in $0.2 \%$ of drug-naive patients from HIV-1 Stanford Database), whose presence correlated with a significant increase in resistance to 3TC and FTC; a remarkable increase in resistance was observed also for TDF, though it remained not significant. In addition, our docking simulations show the ability of this mutation to affect the binding affinity of the above-mentioned drugs to the RT enzyme. This structural result is consistent with a previous study showing that T69I confers a selective advantage during chemotherapy with 3TC, presumably by narrowing the template-primer binding cleft and the $2^{\prime}$ deoxynucleoside triphosphate-binding pocket. ${ }^{39}$

Of note, our study highlights that other mutations, localized in the connection domain of HIV-1 RT, can be selected under 3TC/FTC pressure. This is the case of P345S mutation selected under the pressure of $3 \mathrm{TC}+\mathrm{TDF}$. This mutation (occurring in $0.2 \%$ of drug-experienced and completely absent in drug-naive patients from HIV-1 Stanford Database) has been shown to strongly hamper the binding affinity of both NRTIs to RT. Thus, although T69I and P345S are rare mutations in vivo, overall findings support their role in resistance to NRTI.

In addition, other mutations, such as R356K and S379G, selected in the connection and RNAse-H domain of HIV-1 RT (in our in vitro selection experiments) are natural polymorphisms in non-B subtypes [present in of $49.4 \%$ and $4.9 \%$ of HIV-1 non-B subtypes-infected drug-naive patients, and in $68.0 \%$ and $6.0 \%$ of HIV-1 non-B subtypes-infected drugexperienced patients (data from HIV-1 Stanford Database)], thus suggesting that their presence could potentially modulate the virological response to $3 \mathrm{TC}+\mathrm{TDF}$ or FTC + TDF, without a necessary M184V development. Further studies are necessary to verify this hypothesis.

In our in vitro selection experiments, we did not observe the selection of the K65R under the pressure imposed by tenofovir. This can be explained by the fact that we have used the HIV-1 B subtype laboratory strain HIV-1IIIB. Previous studies have shown a slower K65R emergence in HIV-1 B subtype due to the requirement of 2 nucleotide passages from the Lysine $(\mathrm{K})$ to Arginine $(\mathrm{R}) .^{40}$ It is conceivable that the in vitro K65R selection in HIV-1 B subtype requires higher concentration of tenofovir as observed in the study by Stone et al. ${ }^{34}$

In conclusion, our study shows lower rates of M184V development in FTC + TDF regimens versus 3TC + TDF, and suggests a potential role of boosted PIs and TDF in delaying the M184V emergence. Novel RT mutational patterns, more 
complex than currently known, can contribute to resistance to NRTI, including FTC/3TC. These results may have implications in selecting NRTIs both at the time of starting HAART and at therapeutic failure.

\section{ACKNOWLEDGMENTS}

We thank Andrea Bidittu for data management and also Mrs. Becky Provinciael and Mrs. Nathalie De Keersmaeker for excellent technical assistance.

\section{REFERENCES}

1. Hogg RS, Bangsberg DR, Lima VD, et al. Emergence of drug resistance is associated with an increased risk of death among patients first starting HAART. PLoS Med. 2006;3:e356.

2. Kozal MJ, Hullsiek KH, Macarthur RD, et al, and Terry Beirn Community Programs for Clinical Research on AIDS (CPCRA). The Incidence of HIV drug resistance and its impact on progression of HIV disease among antiretroviral-naïve participants started on three different antiretroviral therapy strategies. HIV Clin Trials. 2007;8:357-370.

3. Zaccarelli M, Tozzi V, Lorenzini P, et al, and Collaborative Group for Clinical Use of HIV Genotype Resistance Test (GRT) at National Institute for Infectious Diseases Lazzaro Spallanzani. Multiple drug class-wide resistance associated with poorer survival after treatment failure in a cohort of HIV-infected patients. AIDS. 2005;19:1081-1089.

4. Zaccarelli M, Tozzi V, Lorenzini P, et al. The V118I mutation as a marker of advanced HIV infection and disease progression. Antivir Ther. 2007;12: 163-168.

5. Gathe JC Jr, Ive P, Wood R, et al. SOLO: 48-week efficacy and safety comparison of once-daily fosamprenavir/ritonavir versus twice-daily nelfinavir in naive HIV-1-infected patients. AIDS. 2004;18:1529-1537.

6. Walmsley S, Bernstein B, King M, et al, and M98-863 Study Team. Lopinavir-ritonavir versus nelfinavir for the initial treatment of HIV infection. N Engl J Med. 2002;346:2039-2046.

7. Gupta SK, Rosenkranz SL, Cramer YS, et al. The pharmacokinetics and pharmacogenomics of efavirenz and lopinavir/ritonavir in HIV-infected persons requiring hemodialysis. AIDS. 2008;22:1919-1927.

8. Margot NA, Enejosa J, Cheng AK, et al, and Study 934 Team. Development of HIV-1 drug resistance through 144 weeks in antiretroviral-naïve subjects on emtricitabine, tenofovir disoproxil fumarate, and efavirenz compared with lamivudine/zidovudine and efavirenz in study GS-01-934. J Acquir Immune Defic Syndr. 2009;52:209-221.

9. Hammer SM, Eron JJ Jr, Reiss P, et al. Antiretroviral treatment of adult HIV infection: 2008 recommendations of the International AIDS SocietyUSA panel; International AIDS Society-USA. JAMA. 2008;300:555-570.

10. Schinazi RF, Lloyd RM Jr, Nguyen MH, et al. Characterization of human immunodeficiency viruses resistant to oxathiolane-cytosine nucleosides. Antimicrob Agents Chemother. 1993;37:875-881.

11. Tisdale M, Kemp SD, Parry NR, et al. Rapid in-vitro selection of human immunodeficiency virus type 1 resistant to 3 '-thiacytidine inhibitors due to a mutation in the YMDD region of reverse transcriptase. Proc Natl Acad Sci U S A. 1993;90:5653-5656.

12. Johnson VA, Brun-Vézinet F, Clotet B, et al. Update of the drug resistance mutations in HIV-1: December 2009. Top HIV Med. 2009;17:138-145.

13. Kavlick MF, Shirasaka T, Kojima E, et al. Genotypic and phenotypic characterization of HIV-1 isolated from patients receiving (-)-2',3'dideoxy-3'-thiacytidine. Antiviral Res. 1995;28:133-146.

14. Schuurman R, Nijhuis M, van Leeuwen R, et al. Rapid changes in human immunodeficiency virus type 1 RNA load and appearance of drugresistant virus populations in persons treated with lamivudine (3TC). $J$ Infect Dis. 1995;171:1411-1419.

15. Ceccherini-Silberstein F, Erba F, Gago F, et al. Identification of the minimal conserved structure of HIV-1 protease in the presence and absence of drug pressure. AIDS. 2004;18:F11-F19.

16. Balzarini J, Cahard D, Wedgwood O, et al. Marked inhibitory activity of masked aryloxy aminoacyl phosphoramidate derivatives of dideoxynucleoside analogues against visna virus infection. J AIDS. 1998;17:296-302.

17. Snoeck J, Riva C, Steegen K, et al. Optimization of a genotypic assay applicable to all human immunodeficiency virus type 1 protease and reverse transcriptase subtypes. J Virol Methods. 2005;128:47-53.
18. HIV Drug Resistance Database. Available at: http://www.hivdbstanford. edu. Accessed June 2009.

19. Protein Data Bank. Available at: http://www.rcsb.org/PDB. Accessed June 2009.

20. Huang H, Chopra R, Verdine GL, et al. Structure of a covalently trapped catalytic complex of HIV-1 reverse transcriptase: implications for drug resistance. Science. 1998;282:1669-1675.

21. McDonald DQ, Still WC. AMBER torsional parameters for the peptide backbone. Tetrahedron Lett. 1992;33:7743-7746.

22. Still WC, Tempczyk A, Hawley RC, et al. Semianalytical treatment of solvation for molecular mechanism and dynamics. J Am Chem Soc. 1990; 112:6127-6129.

23. Mohamadi F, Richards NGJ, Guida WC. MacroModel: an integrated software system for modeling organic and bioorganic molecules using molecular mechanics. J Comput Chem. 1990;11:440-467.

24. Morris GM, Goodsell DS, Halliday RS, et al. Automated docking using a Lamarckian genetic algorithm and an empirical binding free-energy function. J Comput Chem. 1998;19:1639-1662.

25. DeLano WL. The PyMOL Molecular Graphics System. San Carlos, CA: DeLano Scientific; 2002. Available at: http://www.pymol.org.

26. Balzarini J, Holy A, Jindrich J, et al. Differential antiherpesvirus and antiretrovirus effects of the $(\mathrm{S})$ and $(\mathrm{R})$ enantiomers of acyclic nucleoside phosphonates: potent and selective in-vitro and in vivo antiretrovirus activities of (R)-9-(2-phosphonomethoxypropyl)-2,6-diaminopurine. Antimicrob Agents Chemother. 1993;37:332-338.

27. Coleman RS, Woodward RL, Hayes AM, et al. Dependence of DNA sequence selectivity and cell cytotoxicity on azinomycin A and B epoxyamide stereochemistry. Org Lett. 2007;9:1891-1894.

28. Maserati R, De Silvestri A, Uglietti A, et al, and ARCA Study Group. Emerging mutations at failure of HAART containing lamivudine/ tenofovir or emtricitabine/tenofovir: The ARV Resistance Cohort Analysis Database. Presented at: 16th Conference on Retroviruses and Opportunistic Infections. February 8-11, 2009; Montreal, Canada. Abstract 644

29. Saag MS. Emtricitabine, a new antiretroviral agent with activity against HIV and hepatitis B virus. Clin Infect Dis. 2006;42:126-131.

30. Feng JY, Shi J, Schinazi RF, et al. Mechanistic studies show that (-)-FTC-TP is a better inhibitor of HIV-1 reverse transcriptase than 3TC-TP. FASEB J. 1999; 13:1511-1517.

31. Cammack N, Rouse P, Marr CL, et al. Cellular metabolism of (-) enantiomeric 2'-deoxy-3'-thiacytidine. Biochem Pharmacol. 1992;43:2059-2064.

32. Wang LH, Begley J, St. Claire RL III, et al. Pharmacokinetic and pharmacodynamic characteristics of emtricitabine support its once daily dosing for the treatment of HIV infection. AIDS Res Hum Retroviruses. 2004;20:1173-1182.

33. Bousquet L, Pruvost A, Didier N, et al. Emtricitabine: inhibitor and substrate of multidrug resistance associated protein. Eur J Pharm Sci. 2008;35:247-256.

34. Stone C, Ait-Khaled M, Craig C, et al. Human immunodeficiency virus type 1 reverse transcriptase mutation selection during in vitro exposure to tenofovir alone or combined with abacavir or lamivudine. Antimicrob Agents Chemother. 2004;48:1413-1415.

35. Murry JP, Higgins J, Matthews TB, et al. Reversion of the M184V mutation in simian immunodeficiency virus reverse transcriptase is selected by tenofovir, even in the presence of lamivudine. J Virol. 2003;77:1120-1130.

36. Wainberg MA, Miller MD, Quan Y, et al. In vitro selection and characterization of HIV-1 with reduced susceptibility to PMPA. Antiviral Ther. 1999;4:87-94.

37. Borroto-Esoda K, Vela JE, Myrick F, et al. In-vitro evaluation of the antiHIV activity and metabolic interactions of tenofovir and emtricitabine. Antivir Ther. 2006;11:377-384.

38. Feng JY, Ly JK, Myrick F, et al. The triple combination of tenofovir, emtricitabine and efavirenz shows synergistic anti-HIV-1 activity in-vitro: a mechanism of action study. Retrovirology. 2009;6:44.

39. Sato H, Tomita Y, Ebisawa K, et al. Augmentation of human immunodeficiency virus type 1 subtype E (CRF01_AE) multiple-drug resistance by insertion of a foreign 11-amino-acid fragment into the reverse transcriptase. $J$ Virol. 2001;75:5604-5613.

40. Emerging Mutations at Failure of HAART Containing Lamivudine/ Tenofovir or Emtricitabine/Tenofovir: The ARV Resistance Cohort Analysis Database. Template usage is responsible for the preferential acquisition of the K65R reverse transcriptase mutation in subtype $\mathrm{C}$ variants of human immunodeficiency virus type 1. J Virol. 2009;83:2029-2033. 